\title{
Computer-Aided Diagnosis of Thyroid Nodule: A Review
}

\author{
Deepika Koundal ${ }^{1}$, Savita Gupta ${ }^{1}$ and Sukhwinder Singh ${ }^{1}$ \\ ${ }^{1}$ University Institiute of Engineering \& Technology, Panjab University, Chandigarh, \\ India \\ koundal@gmail.com, savita2k8@yahoo.com, sukhdalip@yahoo.com
}

\begin{abstract}
Diagnostic imaging is an important tool in medical science due to the constrained observations of the expert and uncertainties in medical knowledge. A thyroid ultrasound is a non-invasive imaging study used to detect and classify abnormalities of the thyroid gland. Computerized system is a valuable and beneficial means for feature extraction and classification of thyroid nodule in order to eliminate operator dependency and to improve the diagnostic accuracy. The aim of this paper is to review existing approaches to the automatic classification of nodules in thyroid ultrasound images, highlighting the keypoints and main differences between the used strategies. The aim of this paper is to review existing approaches for the diagnosis of Nodules in thyroid ultrasound images with their performance measures.
\end{abstract}

\section{KEYWORDS}

Thyroid Gland, Nodule, TIRADS, Ultrasound Images, Computer-Aided Diagnosis, Feature Extraction, Classification.

\section{INTRODUCTION}

Thyroid nodule is one of the indicative of thyroid cancer. Nodule can be due to the growth of thyroid cells or a cyst in the thyroid gland. These may be solitary, multiple, or conglomeratic (when some nodules merge into one lesion). Recent studies demonstrated that thyroid nodules can be found in about $66 \%$ of the adult population.

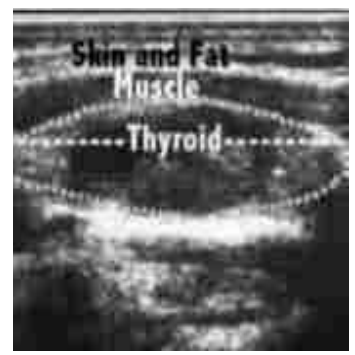

Figure I. Thyroid nodule ultrasound image

The nationwide relative frequency of thyroid cancer among all the cancer cases is $0.1 \%-0.2 \%$ [1]. The incidence of palpable thyroid nodules of the adult population is about $4 \%$ to $8 \%$. As per this statistics, it is concluded that thyroid related cancer is a serious disease which can lead to death, with increasing incidence rates every year. Because of sensitivity and convenience, 
ultrasound is the modality of choice for diagnosis and management of thyroid nodules. A nodule is generally characterized as hypo- echoic, iso-echoic, or hyper-echoic. The echogenicity of a thyroid nodule refers to its brightness compared to the normal thyroid parenchyma. Hypoechogenicity is associated with thyroid malignancy. Most of the thyroid nodules tend to have various internal echogenicities, which makes the definite diagnosis of them difficult. If the characteristic echogenicity for the major components of the thyroid nodule can be realized, the interpretation of thyroid ultrasound would be more realistic, the misdiagnosis rate of thyroid cancer would be decreased and management facilitated. In follicular adenoma, changes are either iso-echoic or hyper-echoic. In carcinoma, the echogenicities vary [2]. Several ultrasound features have been found to be associated with an increased risk of thyroid cancer, including hypo-echogenicity, predominantly solid composition, etc. No ultrasound feature has both a high sensitivity and a high positive predictive value for thyroid cancer [1].

Most of the thyroid nodules are heterogeneous with various internal components, which confuse many radiologists and physicians with their various echo patterns in thyroid nodules. The malignancy of thyroid nodule's can be assessed by Thyroid Imaging Reporting and Data System (TIRADS) categorizing as malignant, suspicious for malignancy, borderline, probably benign and benign [2].

For diagnosing thyroid diseases, Ultrasound (US) and Computerized Tomography (CT) are two of the most popular imaging modalities. Primary detection of nodular lesions, such as nodular goiter and thyroid tumors using US images are well documented in textbooks and many articles $[3,4]$. US imaging is inexpensive, non-invasive and easy to use. Although MRI (Magnetic Resonance Imaging) and CT have clear visualization than US images, but US images are often adopted due to their cost-effectiveness and portability in smaller hospitals. The thyroid is well suited to ultrasound study because of its superficial location, vascularity, size and echogenicity. Malignant thyroid nodules having distinct histopathological components and vague boundaries often merged with surrounding tissues resulting in a difficult task during delineation of nodule's shape [6]. Hence, a novel computerized segmentation and classification method is required to improve the accuracy and to decrease the misdiagnosis rate as early detection is necessary for effective diagnosis. Therefore, some Computer-Aided Diagnosis (CAD) of Thyroid Ultrasound is necessary in order to delineating nodules, classifying benign/malignant and estimating the volumes of thyroid tissues to increase reliability and reduce invasive operations such as biopsy and Fine Needle Aspiration (FNA).

The rest of this paper is organized in three sections. Section 2 describes the CAD for the diagnosis of nodules in thyroid gland. The feature Extraction and Selection techniques are given in section 3.The Classification techniques are given in Section 4. Section 5 consists of Evaluation Performance. Finally, the conclusions as well as future directions are summarized in Section 6.

\section{COMPUTER-AIDED DIAGNOSIS FOR THYROID ULTRASOUND}

Since US is much more operator-dependent, reading US image requires well-trained and experienced radiologists. CAD can minimize the operator-dependent nature inherent in US imaging and make the diagnostic process reproducible. CAD has been investigated to help radiologists in making accurate diagnosis; even well-trained experts may have a high interobserver variation rate. Another advantage of CAD system is that it can obtain some features, such as computational features and statistical features, which cannot be obtained visually and intuitively by medical doctors. Generally, CAD systems for cancer diagnosis involve various stages, as shown in Figure II. It should be noted that research into the use of CAD is not done so with an eye toward eliminating doctors or radiologists, rather the goal is to provide doctors and radiologists a second opinion and help them to increase the diagnosis accuracy, reduce biopsy 
rate and save their time and effort. All these algorithms can be categorized in various types depending on the strategy chosen for classifying nodule.

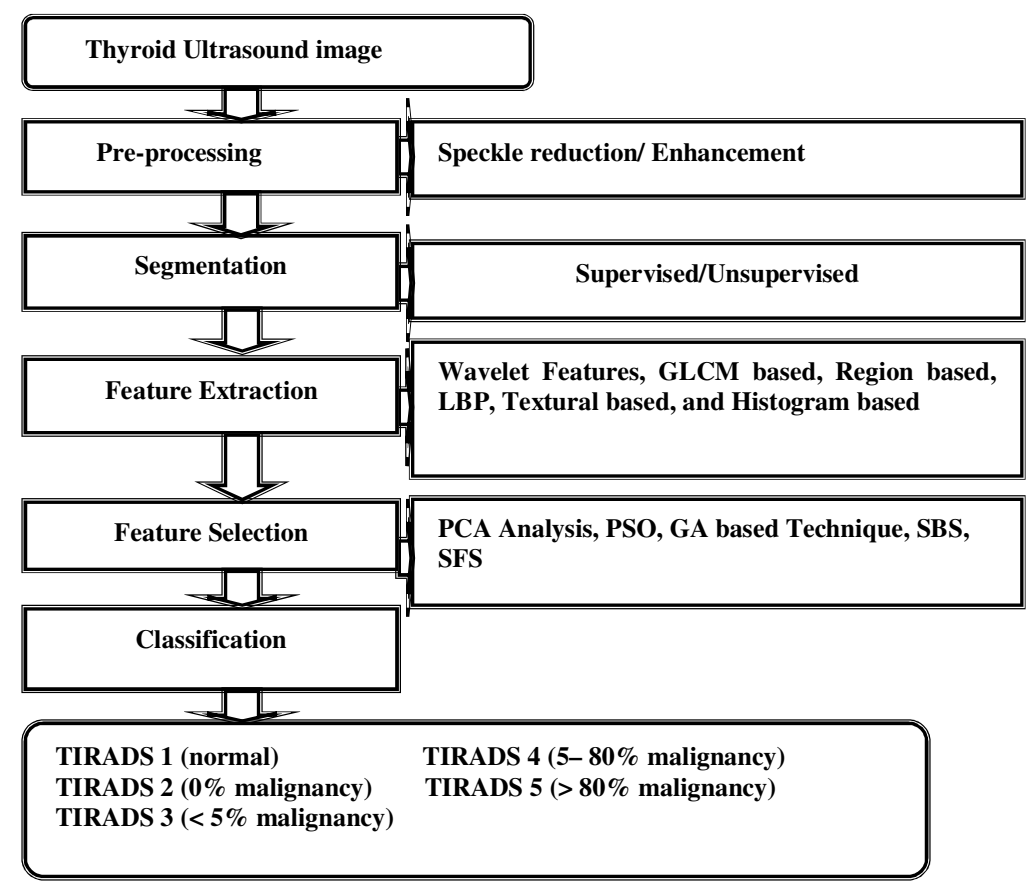

Figure II. Computer-Aided Diagnosis (CAD) System

\section{Feature Extraction \& SELECTION}

Feature extraction is the process of extracting certain characteristic attributes and generating a set of meaningful descriptors from an image. It is used to find a feature set of tissue that can accurately distinguish lesion/non-lesion or benign/malignant. Recently, various feature extraction methods were proposed from which lot of features from medical images can be obtained. However, it is difficult to select significant features from the extracted features. There is no single feature that can accurately determine whether a nodule is benign or malignant. In addition to features that can be derived from the inside of the nodule, the tissue texture around the margin of the nodule is also important. The growth of malignant tumors tends to distort the surrounding tissue texture, while benign nodules tend to have smooth surfaces with more uniform texture around them. Different shapes and margins have different likelihoods of malignancy. Thus, texture features have the potential to capture characteristics that are diagnostically important but are not easily visually extracted.

Feature selection is a process of feature reduction by removing irrelevant, redundant or noisy data and has an immediate effect on application by accelerating the classification algorithm. A typical feature selection process consists of four basic steps: namely, subset generation, subset evaluation, stopping criterion and result validation. Feature selection can be categorized into three classes. The filter model, which uses the general characteristics of the data to evaluate the features and selects the optimal feature subset(s) with no classification or learning algorithm such as methods based on correlation, entropy, mutual information, etc. The wrapper model, which uses learning algorithm and searches for features which improve the learning performance, such as methods based on greedy and genetic algorithms. The hybrid model which combines the above two approaches. The feature space could be very large and complex, so 
International Journal of Computer Science \& Engineering Survey (IJCSES) Vol.3, No.4, August 2012

extracting and selecting the most effective features are very important. Some of the features extracted from thyroid tissue are summarized in Table I.

\begin{tabular}{ll}
\hline REF. & FEATURE EXTRACTION APPROACH \\
\hline$[42][43][44]$ Grey Level Histogram \\
{$[45]$} & Muzzolini 'S Features \\
{$[42][45]$} & Co-Occurrence Matrix \\
{$[11]$} & Radon Transform \\
{$[47]$} & Local Binary Patterns \\
{$[47]$} & Fuzzy Local Binary Pattern \\
{$[7]$} & Mean, Variance, Coefficient Of Local Variation Feature, \\
& Histogram Feature, Normalized Multi Scale, Intensity Different \\
{$[48]$} & NMSID Feature, Homogeneity \\
{$[14]$} & Statistical Pixel Level Features \\
{$[11]$} & Morphology And Tissue Reflectivity \\
{$[48]$} & Textensity And Statistical Textural Feature \\
\hline
\end{tabular}

Table I. Feature Extraction Approaches for Thyroid Ultrasound Image Analysis

Some of the features which are extracted from thyroid tissue are given below:

1) Haar wavelet features: are the significant features for segmentation in US images [7]. Hence, the two statistical features extracted as:

$$
\text { Mean of LL band: } \mu_{x, y}=\frac{1}{M^{2}} \sum_{(x, y) \in B} I(x, y)
$$

$$
\text { Variance of LL band: } \sigma_{x, y}^{2}=\frac{1}{M^{2}} \sum_{(x, y) \in B}\left(I(x, y)-\mu_{x, y}\right)^{2}
$$

Where $I(x, y)$ denotes the intensity of a pixel $(x, y)$ in ROI block which passed through the Haar transformation, and B denotes a block size of $M \times M[8]$.

2) Homogeneity feature: is a major measurement for degree of smoothness. It is a useful information to represent the thyroid gland as thyroid gland is smoother than other regions in the US image. The homogeneity feature homo $o_{x, y}$ of a pixel located at $(x, y)$ is defined as

$$
H o m v_{x, y}=\sqrt{\sigma_{x, y}^{2} \times} \sigma_{x, y}^{2} / \mu_{x y}
$$

Where $\mu_{x y}$ is local mean and $\sigma_{x_{y} y}^{2}$ is local variance of a pixel located at (x,y) with a block size of $M \times M[8]$.

3) Histogram feature: is used to measure texture characteristic of a $M \rtimes M$ block. The value of a histogram feature HF is defined as follows:

$$
\begin{aligned}
& H F=\sum_{i=H-10, I \neq H}^{H 110} h i s t o(i) \\
& H={ }_{i}^{\operatorname{argmax}(h i s t o(i))}
\end{aligned}
$$


Where histo (i) is histogram of a block size of $M \times M$, and $i$ denotes gray level value \pm 10 is a tolerance value[8].

4) Block Difference Of Inverse Probablities Features: BDIP feature is defined as differnce between the number of pixels in a block and ratio of the sum of pixel intensities in the block to the maximum in the block.

$$
\mathrm{BDIP}=M^{2}-\Sigma_{(x, y) \in B} l(x, y) /(x, y) \in \max I(x, y)
$$

Where $I(x, y)$ denotes the intensity of a pixel $(x, y)$, and B denotes a block with size of $M \times M[8]$.

5) Normalized Multiscale Intensity Difference Feature: NMSID feature is defined as differences between the pixel pairs with horizontal, vertical, diagonal and asymmetric diagonal directions[8].

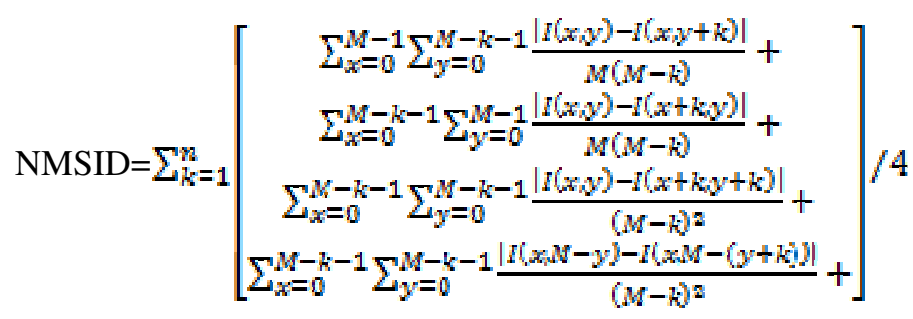

Where $I(x, y)$ denotes the intensity of a pixel $(x, y)$ in a block size of $M \times M$, and $n$ denotes maximum horizontal or vertical distance.

6) Coefficient of local variation feature: $\mathrm{CV}$ is a normalized measure of dispersion of a probability distribution. Because the texture of thyroid glands differs from those of other regions in the US image, $\mathrm{CV}$ is a useful index to represent it.

$$
L C V_{x y}=\frac{\sigma_{N_{y} y}}{\mu_{x_{y}}}
$$

7) Textural Features: Texture patterns appearing in US images can be represented by a fuzzy distribution of Local Binary Patterns, referred to as Fuzzy Loc al Binary Patterns (FLBP) features [9]. Although the original approach of Local Binary Pattern (LBP) [10] has been used successfully in many studies [11], it has also been proven to be sensitive to small variation s of the pixel intensities usually caused by noise. The FLBP is an enhanced extension of the LBP approach, capable of better coping with speckle noise [9], a common characteristic of all US images [12].

8) Intensity Features: In US images a substantial amount of information concerning the pathology of the examined tissue is contained in image echogenity [13]. Several studies on US medical images have been using echogenity features based on grey-level histograms (GLH) [14]. In the proposed system, fuzzy grey-level histograms (FGLH) have been utilized for intensity representation, given that they are well known for their insensitivity to noise [15].

9) Morphological features: describe the shape and the boundary regularity of each nodule and comprised several 1st order statistics of the boundary radius along with area, smoothness, concavity, and symmetry and fractal dimension. The radius represents the Euclidean distance from the nodule's centroid to each of the boundary points [16].

10) Wavelet local maxima features: The generation of the wavelet local maxima features has been employed in the edge map from the last wavelet decomposition before and after the 
speckle reduction procedure. The edge map that includes all detected Local Maxima (LM) and the edge map that includes only those LM that correspond to important. Various 1st order statistics features from the LM histogram are computed such as mean value, standard deviation, entropy, kurtosis, etc.

11) Rayleigh distribution parameter: The speckle pattern present in the ultrasound images is a result of the interference of echoes at the surface of the transducer, which emanate from the acoustic impedance of the tissues. One of the most used in ultrasound (US), LASER and Synthetic Aperture Radar (SAR) is the Rayleigh distribution used to describe this kind of pattern. Another view of the problem is to accurately reconstruct the ultrasound images to provide a measure of the local acoustic impedance of the tissues. Let $\mathrm{X}=\left[x_{i, j}\right\}$ and $\mathrm{Y}=\left\{y_{i, j}\right\}$ be a

$N \times M$ image presenting the acoustic impedance of the tissue and a speckle image, respectively. The speckle pattern of the image $\mathrm{Y}=\left\{y_{i j}\right\}$ is described by a Rayleigh distribution,

$$
p\left(y_{i, j} \mid x_{i, j}\right)=\frac{y_{i j}}{x_{i, j}} e^{-\frac{y_{i, j}{ }^{n}}{2 x_{i j}}}
$$

The estimation of $\mathrm{X}$ from $\mathrm{Y}$ is formulated as the following optimization task

$$
\bar{X}=\frac{\operatorname{argmin} E(X, Y),}{X}
$$

Where $F(X, Y)$ is an energy function.

12) Randon Transform Features: It is observed that the thyroid tissue may be characterized by different directionality patterns observed in the ultrasound images. The encoding of the directional patterns is realized by means of Randon Transform features[13]. The idea is to project the image intensity along a radial line oriented at different angles $(0,45,90$ and 135 degrees).

13) Linear Binary Pattern: LBP approach provides a theoretically and computationally simple an efficient methodology for texture analysis based on the concept of texture primitives, often called texels or textons. The LBP operator was originally designed for texture description. The operator assigns a label to every pixel of an image by thresholding the $3 \times 3$-neighborhood of each pixel with the center pixel value and considering the result as a binary number. Then the histogram of the labels can be used as a texture descriptor. The textural characteristics of the thyroid tissue are encoded by histograms of Local Binary Patterns (LBP) [17]. The advantages of this novel scheme include increased accuracy in nodule detection, compared with the conventional ultrasound image analysis methods, and time efficiency. The various feature extraction approaches for thyroid nodule in ultrasound images are given in [28].

In [10], 78 textural features are extracted from ROIs, which were outlined by radiologist and recognized by biopsy. Among them 13 features extracted from the co-occurrence matrix are correlation, difference entropy, difference variance, sum average, sum entropy, sum of squares, sum variance, contrast, energy, entropy, local homogeneity, cluster shade, and cluster prominence. The dissimilarity extracted from the statistical feature matrix is used for statistical analysis. Five features short runs emphasis, long runs emphasis, gray level uniformity, run length uniformity, and run percentage are calculated from the gray level run-length matrix. The features LE mean, EL mean, SL mean, EE mean, LS mean, LE variance, EL variance, SL variance, EE variance, and LS variance are calculated from Laws; texture energy measures. The features small number emphasis, large number emphasis, number non-uniformity, second moment, and entropy are calculated from neighboring gray level dependence matrix. The mean, standard deviation, and Laws; features of the LL sub-band image are calculated as wavelet features. Finally, the mean and standard deviation of the magnitude and phase angle of local Fourier coefficients maps are computed as textural feature. They are means of 8 magnitudes, are 
means of 8 phase angles, are standard deviations of 8 magnitudes, and are standard deviations of 8 phase angles.

In $[12,17]$ a set of novel fuzzy features for thyroid US texture representation, demonstrating noise-resistant properties are presented, analyzed and evaluated. The textural feature extraction scheme is based on the fuzzification of the LBP approach. The results have provided an accurate representation of the thyroid texture.

In 2009, Saiti et al.[16] argued feature selection as an important problem via diagnosis and demonstrate that GAs (Genetic Algorithms) provide a simple, general and powerful framework for selecting good subsets of features leading to improved diagnosis rates. The proposed methods are hybrid technique consisting of SVM with Genetic algorithm and PNN with GA. The classification accuracy of PNN with GA feature selection method is $96.8 \%$, sensitivity is $99.02 \%$, specificity is $84.2 \%$ and classification accuracy of SVM with GA feature selection is $93.33 \%$, sensitivity is $97.9 \%$ and specificity is $99.7 \%$. In this study sequential forward selection (SFS), sequential backward selection (SBS) and Genetic Algorithm are used as feature selection methods. In the second stage, SVM and PNN are used to classify thyroid data.

In [19], the physician manually extracted 2n ROIs with size of $M \times M$ ( $n$ thyroid ROIs and $n$ non-thyroid ROIs) from the suspicious thyroid region. Six textural features are extracted from the selected ROIs. These textual features including Haar wavelet features, homogeneity feature, histogram feature, block difference of inverse probabilities (BDIP) and normalized multi-scale intensify difference (NMSID) will be used in the RBF neural network to classify the thyroid region. A wrapper-type method is carried out for feature selection. This method introduces a classifier to show us which combination of features has the best performance, i.e., the highest accuracy of classification that using this combination. Five binary-SVMs are used to perform the feature selection.

In [20], a number of textural features were automatically calculated from the segmented Region of Interest (ROI) of each thyroid nodule. Textural features are related to the gray-tone structure of the thyroid nodule as depicted on the ultrasound unit, and carry information relevant to the risk factor of malignancy. Four features were computed from the nodule's gray-tone histogram, 26 from the co-occurrence matrix and 10 from the run-length matrix (Galloway 1975). The selection of best feature combination that will maximize the performance of the classifier is necessary but time-consuming and computationally demanding procedure. The exhaustive search method involved designing the classifier by means of every possible feature-combination and all thyroid data available, each time testing the classifier's performance in correctly classifying the thyroid data, and finally selecting that feature combination that demonstrated the highest classification accuracy with the smallest number of textural features. The exhaustive search method was chosen instead of statistical methods such as F-statistics because the latter could result in unreliable error probability estimation, because of the small size of the data set.

In [21], six rectangular windows were extracted from each lobe after segmenting the thyroid gland. Then three different types of features Rayleigh parameter, 3 wavelet energy coefficients and 4 randon transform parameters are computed from each rectangular window which is also combined with longitudinal mid-distance measure for each thyroid gland.

In [23], a set of twenty Morphological features (mean radius, radius entropy, radius standard deviation, parameter, area, circularity, smoothness, convex hull mean radius, concavity, number of concave points, symmetry, fractal dimension) and wavelet local maxima (first order histogram, mean value, entropy, $3^{\text {rd }}$ degree central moment, kurtosis, skewness, variance, standard deviation) features are generated from segmented thyroid. The boundary of each thyroid nodule was extracted through a hybrid multi-scale model. This model integrated in a cascade level at first a speckle reduction edge detection procedure that employed dyadic wavelet transform and local maxima regularity estimation. Consequently, a multi-scale structure model for boundary detection and finally the Hough transform for the boundary nodule extraction. 
In [27], the feature extraction and the feature reduction processes are performed in feature reduction stage of the GDA-WSVM expert system for diagnosis of thyroid diseases. For this goal, the thyroid gland dataset was composed. The dimension of this thyroid gland dataset, which has five features, was reduced to three features using Generalized Discriminant Analysis (GDA).

\section{Classification}

The suspicious regions will be classified as lesion/non-lesion or benign/malignant based on the selected features by various classification methods. The Thyroid Imaging Reporting and Data System (TIRADS) are a standardized US characterization and reporting data system of thyroid lesions for clinical management. The TIRADS is based on the concepts of the Breast Imaging Reporting Data System (BIRADS) of the American College of Radiology [2]. The categories are as follow:

- TIRADS 1: normal thyroid gland.

- TIRADS 2: benign conditions ( $0 \%$ malignancy).

- TIRADS 3: probably benign nodules ( $<5 \%$ malignancy).

- TIRADS 4: suspicious nodules (5-80\% malignancy rate). A subdivision into 4a (malignancy between 5 and 10\%) and $4 \mathrm{~b}$ (malignancy between 10 and 80\%) was optional.

-TIRADS 5: probably malignant nodules (malignancy $>80 \%$ ).

- TIRADS 6: included biopsy proven malignant nodules.

There are different neural networks used in image segmentation such as Back Propagation neural network, Hopfield neural networks and Self-Organizing Maps (SOM) [24, 25]. Various previous studies based on classifiers used to identify the malignancy in the thyroid lesion are mentioned in [29, 30]. Many machine learning techniques such as Linear Discriminant Analysis (LDA), Support Vector Machine (SVM) and Artificial Neural Network (ANN) have been studied for thyroid lesion classification [31]. The classification accuracy of various classifiers for thyroid nodule in ultrasound images are summarized in table II.

An approach for differentiating benign and malignant thyroid nodules based on SVM with biased penalties is presented by Ma et al.[8]. The Leave-One-Out Cross-Validation (LOOCV) is conducted in evaluating the performance of classification method. The goal was to obtain the Maximum Geometric Mean (MGM) of sensitivity and specificity. The results showed that the method is able to get $90.1 \%$ with the sensitivity of $93.8 \%$ and the specificity of $86.6 \%$.

In [10], five support vector machines (SVM) were adopted to select the significant textural features and to classify the nodular lesions of thyroid. A lot of texture extraction methods were used to characterize the thyroid nodules. Accordingly, the thyroid nodules could be classified by the corresponding textural features. The support vector machine (SVM) has the capability of generating a hyper-plane to separate two sets and providing good generalization performance. Firstly, 78 textural features are extracted from ROIs, which were outlined by radiologist and recognized by biopsy to recognize these thyroid nodules. To select the significant features, the SVMs are applied for feature selection and obtain the more discriminative feature set of different categorizes of thyroid nodules. Finally, each SVM was trained by the shifted features of corresponding category. The SVMs are then applied to select the significant features from the extracted 78 features. Each SVM is trained by specific features, having more discrimination between two types of thyroid nodules. A 3-fold cross validation method is adopted, i.e., the ratio of training and test data is $2: 1$. For the generalization, the validation is performed 10 times. The average accuracies are all higher than $96 \%$. To provide a helpful way to decrease the erroneous diagnosis, a SVM-based thyroid nodules classification method was proposed. The comparison results demonstrated that the capability of feature selection of the proposed method was similar 
to the Sequential Floating Forward Selection (SFFS) method. However, the proposed method is faster than the SFFS method. The experimental results showed the proposed classification approach successfully identified six kinds of thyroid nodules with good performance. These results are very helpful in interpretation of thyroid ultrasound and in enhancement of diagnostic performance of ultrasound guided needle aspiration.

A comparative thyroid disease diagnosis realized by multilayer, probabilistic, and learning vector quantization neural networks is presented by Temurtas [11]. The Classification accuracy of Multilayer Neural Networks (MLNN) with LM $(3 \times$ FC) is 92.96 , PNN $(3 \times$ FC) is $94.43 \%$, LVQ $(3 \times$ FC) is $89.79 \%$, MLNN with LM $(10 \times$ FC) is $93.19 \%$, PNN $(10 \times$ FC) is $94.81 \%$, LVQ $(10 \times$ FC) is $90.05 \%$. The results showed that Probabilistic Neural Network has given the best classification accuracies for thyroid disease dataset.

In 2005, Tsantis et al. [20] proposed a computer-based image analysis system employing the SVM classifier for the automatic characterization of 120 verified thyroid nodules into two main classes, high-risk and low-risk for malignancy. The system performance of SVM was compared with the Quadratic Least Squares Minimum Distance classifier (QLSMD) and Quadratic Bayesian (QB) classifier for assessing thyroid nodule malignancy risk on US images. Here the accuracy of SVM in classifying the low and high risk nodules is $96.7 \%$ where QLSMD classifier is $92.5 \%$ and QB classifier is $92 \%$.

\begin{tabular}{|c|c|c|c|}
\hline $\begin{array}{l}\text { Publication } \\
\text { year }\end{array}$ & Ref. & Method & Accuracy $(\%)$ \\
\hline \multirow[t]{4}{*}{1984} & [42] & FA FA+C4.5 (Pruned) & 94.38 \\
\hline & & FAFA+C 4.5 (Rules) & 94.38 \\
\hline & & Einstein & 91.91 \\
\hline & & FAF A+Einstein & 93.34 \\
\hline 1997 & [40] & A Fuzzy Classifier with Ellipsoidal Regions & 93.34 \\
\hline \multirow[t]{4}{*}{1997} & {$[41]$} & MLP & 36.74 \\
\hline & & LVQ & 81.86 \\
\hline & & RBF & 72.09 \\
\hline & & PPFNN & 78.14 \\
\hline \multirow[t]{3}{*}{1999} & [39] & k-NN method & 96.90 \\
\hline & & EACH method & 95.60 \\
\hline & & RPA method & 96.10 \\
\hline \multirow[t]{2}{*}{2002} & {$[30]$} & 3NN-Par & 94.20 \\
\hline & & FED IC-Plain & 96.10 \\
\hline \multirow[t]{2}{*}{2006} & {$[31]$} & EDA & 98.06 \\
\hline & & WEDA & 98.00 \\
\hline \multirow[t]{2}{*}{2006} & {$[32]$} & HMM method & 87.91 \\
\hline & & SOM method & 88.84 \\
\hline \multirow[t]{4}{*}{2006} & {$[33]$} & LDA & 93.44 \\
\hline & & SVM & 94.44 \\
\hline & & GPC-EP (s-soft) & 96.75 \\
\hline & & GP C-EP(m-soft) & 97.23 \\
\hline 2007 & [29] & AIRS & 81 \\
\hline
\end{tabular}


International Journal of Computer Science \& Engineering Survey (IJCSES) Vol.3, No.4, August 2012

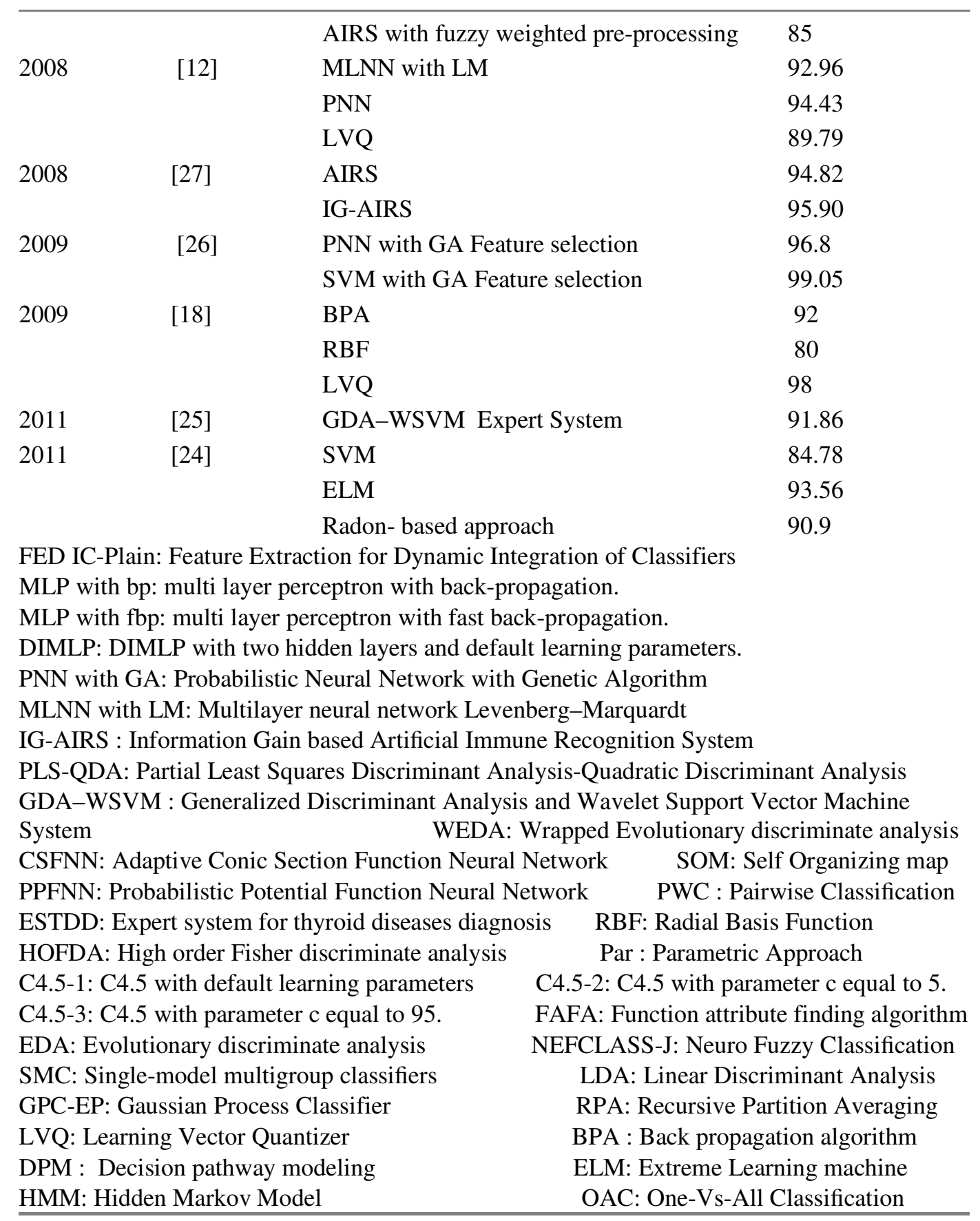

Table II. Accuracy of Thyroid Classifiers

Zhang et al. in 1998 [32] diagnoses the thyroid disease by training a neural network on the basis of signs and symptoms that outperforms human physicians especially in the presence of noise. This is probably due to the fact that human physicians only consider a limited number of signs and symptoms in their diagnosis, whereas the neural network takes all input into consideration and due to the inherent noise tolerant nature of neural network. An 84-14-12 neural network is implemented using 84 signs and symptoms of thyroid diseases as input and the 12 kinds of thyroid illness as output. The training takes place first by varying the number of hidden nodes, then by varying the number of hidden layers, the learning coefficient, the momentum 
coefficient, and the noise inject and the tolerance between output and targets. The training is terminated when the neural network can diagnose all the targeted diseases.

In another work by Savelonas et al. [33], the potential of boundary descriptors for the assessment of thyroid nodules on US images is investigated according to malignancy risk. The classification experiments are performed by the $\mathrm{NN}$ classifier for feature vectors derived from all combinations of compactness, chain code histogram and fractal dimension. The experimental evaluation on real thyroid US images lead in discriminating between medium-risk and high-risk nodules, with an area under the curve are 0.93 . The study is limited to cases of hypo-echoic nodules, since hypo-echogenicity in conjunction with boundary irregularity demonstrate an extremely high correlation with malignancy risk. A k-nearest neighbor (k-NN) classifier was used for the classification. The value of $\mathrm{k}$ was experimentally determined as $\mathrm{k}=5$.

In 2009, the diagnosis of thyroid producing thyroid disorders using ANNs is presented by Shukla et al.[34]. The BP network model has 40 hidden neurons and accuracy of $92 \%$. Here, the diagnosis is 46 out of 50 cases. The Radial Basis Function (RBF) network model has spread value $0.15,100$ hidden neurons and accuracy of $80 \%$ for diagnosis of thyroid disorders. Here, the diagnosis is correct in 40 out of 50 cases. The network with 28 hidden neurons and an accuracy of $98 \%$ is the best Linear Vector Quantizer (LVQ) Network. Here, the diagnosis is correct in 49 out of 50 cases. On comparison of the performance of three neural network architectures, LVQ Network is found to have the best accuracy of diagnosis, which is $98 \%$.

Rouhani et al. [35] used several ANN architectures RBF, Probabilistic Neural Network (PNN) and Linear Vector Quantizer (LVQ) and SVMs for diagnosing thyroid diseases. As the degree of disease development is a critical parameter in medical treatment, networks are designed to classify the grade of diseases, too. The authors found out that the RBF network and PNN outperforms other network including GRNN, LVQ, and SVM. In three cases (Hypothyroid, Normal, and subclinical hypothyroid) the RBF network has the best accuracy. The PNN has the performance for three cases (Hypothyroid, Hyperthyroid, and subclinical hyperthyroid), too. For the later two cases, the GRNN has the same good performance and the SVM has the best performance in diagnosis normal function of thyroid gland. The overall accuracy of diagnosis system is range from near $96 \%$ to $99 \%$.

\subsection{Artificial Immune System}

Artificial Immune Systems (AIS) is a term to adaptive systems, emerging in 1980's, that extract ideas and metaphors from the biologic immune system to solve computer problems (Castro, 2007). This framework of immunologic engineering is composed of a representation of the system's components, a set of mechanisms to evaluate the interaction between individuals and their environment. The environment is simulated by a series of stimuli (input patterns), one or more evaluation functions (fitness) and adaptive procedures rule the system dynamics, which is, how its behavior changes over the time.

In 2007, the thyroid disease with a new hybrid machine learning method was diagnosed by Polat et al. [36]. By hybridizing AIRS (Artificial Immune Recognition System) with a developed Fuzzy weighted pre-processing, a method is obtained to solve the diagnosis problem via classifying. The robustness of AIRS with Fuzzy weighted pre-processing method with regard to sampling variations is examined using a 10-fold CV method and obtained a classification accuracy of $85 \%$. The classification accuracy of $81 \%$ was obtained with AIRS classification system.

A new Artificial immune system named the Information Gain based Artificial Immune Recognition System (IG-AIRS) is proposed by Kodaz et al. [37]. The proposed system involves two stages. At the first stage, information gain values of data attributes are obtained. Then, at the second stage, these values are applied to the Artificial Immune Recognition System (AIRS) with information gain based Euclidean distance. At the same time, a diagnostic system leading 
to more effective usage of the thyroid disease diagnose is presented. In many AIRS algorithms, the shape-space representation is used to represent the immune system with system units and this representation scheme also defines the interactions of the system units with the environment by means of distance criteria. In the distance-based classification systems, the "curse of dimensionality" problem appeared those that were used in other AIS algorithms. One of the future works related to this system is improving the attribute weighting procedure such that the algorithm performs very well in a great variety of data sets. Besides, the attribute weighting scheme can be applied to other AIRS algorithms that use shape-space representation and performance of these algorithms can be improved in this way. The classification accuracy is 95.90\% with 10-fold CV method.

\subsection{Hybrid Techniques}

In 2007, Savelonas et al. [9] has made some improvement on his previous work by investigating computational characterization of thyroid tissue using supervised classification of directionality patterns in thyroid US images. These patterns may not be always distinguishable by the human eye because of the dominant image noise. The encoding of the directional patterns in the thyroid US images is realized by means of Radon Transform features. The proposed approach achieved higher classification accuracy than the co-occurrence based approach, in the classification of nodular and normal thyroid tissues, as well as in the differentiation between types of thyroid nodules with respect to their malignancy risk. Classification was performed by means of the Leave One Out method. The overall classification accuracy obtained by the application of the proposed Radon-based approach was $84.1 \%$, whereas the classification accuracy obtained by the application of the co-occurrence based approach was 71.2\%. Both approaches outperformed mean intensity on the same dataset, as the classification accuracy obtained with the latter was $67.4 \%$. The overall classification accuracy obtained by the application of the proposed Radonbased approach was $90.9 \%$ for the classification of normal and nodular thyroid tissue with the use of the k-Nearest Neighbor classifier $(\mathrm{k}=5)$. These experiments resulted in classification accuracies of $86.3 \%$ and $74.2 \%$ for the proposed Radon-based approach, and the co-occurrence based approach respectively. The classification accuracy obtained by the application of mean intensity is $75.6 \%$, more than $10 \%$ lower than the one obtained by the proposed approach in classifying malignancy of nodular tissue. The overall classification accuracy obtained by the application of the proposed Radon-based approach was $89.4 \%$.

In [21], a biometric system based on features extracted from the thyroid tissue accessed through 2D US was proposed by Seabra et al. in 2010. A biometric system based on features extracted from the thyroid tissue accessed through 2D ultrasound was proposed. Tissue echo-morphology, which accounts for the intensity echogenicity, texture and structure, has started to be used as a relevant parameter in a clinical setting. In this paper, features related to texture, morphology and tissue reflectivity are extracted from the ultrasound images and the most discriminant ones are selected as an input for a prototype biometric identification system. Several classifiers were tested, with the best results $(90 \%$ identification rate) being achieved with the maximum a posteriori classifier. Several classifiers were tested, with the best results being achieved by a combination of classifiers (k-Nearest Neighbors, MAP and entropy distance). Using leave-one-out cross-validation method the identification rate was up to $94 \%$. Features related to texture and echogenicity were tested individually with high identification rates up to $78 \%$ and $70 \%$ respectively. This suggests that the acoustic impedance (reflectivity or echogenicity) of the tissue as well as texture are feasible parameters to discriminate between distinct subjects. This paper shows the effectiveness of the proposed classification, which can be used not only as a new biometric modality but also as a diagnostic tool.

In 2011, Selvathi et al.[22] developed an automatic system that classified the thyroid images and segmented the thyroid gland using machine learning algorithms. The classifiers such as SVM, ELM are used. The features such as mean, variance, Coefficient of Local Variation 
Feature, Histogram Feature, NMSID Feature, and Homogeneity are extracted and these features are used to train the classifiers such as ELM and SVM. It is observed that the segmentation using ELM is better than SVM classifier. In this, two modules were proposed to develop a computer aided diagnosis system of thyroid ultrasound images. In the first module, the thyroid ultrasound images are classified as normal (thyroid gland with modules) using features such as mean, variance, coefficient of local variation, histogram, homogeneity and SVM classifier. From the first module classified result, the thyroid gland regions are segmented from the normal region in the normal thyroid images using ELM and the performance are compared with SVM. The results obtained are compared with ground truth obtained from radiologist and the performance measures such as accuracy are calculated. The performance of classification can be measured by means of accuracy, specificity, sensitivity and precision. The accuracy of SVM has 84.7\%, ELM has 93.56\% and the proposed method has 100\%.

In 2008, Keles et al. [26] aimed at diagnosing thyroid diseases with an expert system named as an ESTDD (Expert System for Thyroid Disease Diagnosis). The fuzzy rules are found by using neuro-fuzzy method, which will be emplaced in proposed system. This system could diagnose with $95.33 \%$ accuracy of thyroid diseases.

\section{Evaluation}

\begin{tabular}{|c|c|c|c|}
\hline REF. & MEASURE & DEFINITION & PARAMETER \\
\hline \multirow{4}{*}{ [29] } & True positive & $\mathrm{TP}(\%)=\frac{\left|A_{m} \cap A_{n}\right|}{\left|A_{n}\right|}$ & \multirow{4}{*}{$\begin{array}{l}\text { Where, } A_{m} \text { refers to the } \\
\text { tumor area determined by a } \\
\text { group of radiologists and } A_{n} \\
\text { is the area determined by the } \\
\text { new algorithm. }\end{array}$} \\
\hline & False positive & $\mathrm{FP}(\%)=\frac{\left|A_{m} \cup A_{n}-A_{n}\right|}{\left|A_{m}\right|}$ & \\
\hline & $\begin{array}{l}\text { Similarity } \\
\text { index }\end{array}$ & $\mathrm{SI}(\%)=\frac{\left|A_{m} \cap A_{n}\right|}{\left|A_{m} \cup A_{n}\right|}$ & \\
\hline & False negative & $\mathrm{FN}(\%)=1-\mathrm{TP}(\%)=\frac{\left|A_{m} \cup A_{n}-A_{m}\right|}{|A m|}$ & \\
\hline \multirow{5}{*}{$\begin{array}{l}{[38]} \\
{[22]}\end{array}$} & Accuracy & $=\left(A_{T P}+A_{T N}\right)$ & \multirow{5}{*}{$\begin{array}{l}\text { Where, } A_{P} \text { is the total no. of } \\
\text { positive pixels, } A_{N} \text { is the } \\
\text { total no. of negative pixels, } \\
A_{T P} \text { is the no. of pixels in the } \\
\text { thyroid gland region which } \\
\text { were segmented as thyroid } \\
\text { and } A_{T N} \text { is the no. of pixels } \\
\text { in non-thyroid region which } \\
\text { were segmented as non- } \\
\text { thyroid }\end{array}$} \\
\hline & Sensitivity & $=A_{T P} / A_{P}$ & \\
\hline & Specificity & $=A_{T N} / A_{N}$ & \\
\hline & $\begin{array}{l}\text { Positive } \\
\text { predictive value }\end{array}$ & $P P V=A_{T P} /\left(A_{T P}+A_{F P}\right)$ & \\
\hline & $\begin{array}{l}\text { Negative } \\
\text { predictive value }\end{array}$ & $\begin{array}{c}N P V=A_{T N} /\left(A_{T N}+A_{F N}\right) \\
A_{F P}=A_{N}-A_{T N} \text { and } A_{F N}=A_{P}-A_{T P}\end{array}$ & \\
\hline
\end{tabular}

Table III. Performance Measures

The performance measures play an important role in evaluating the quality of classification techniques for diagnosis purpose. The quality of an image is examined by objective evaluation as well as subjective evaluation. However, as per literature, there are various metrics used for evaluating classification methods in general or for specific application. Some of the metrics used for measuring the thyroid image classification techniques are shown in Table III. 


\section{Conclusion AND DiscuSSION}

Various techniques are applied by different researchers to process Thyroid US as many of the structures are hardly visible due to noise ambiguity, vagueness and uncertainty in TUS. In order to detect the abnormal structure, intuitive ways must be found out to interpret and describe the inherent ambiguity and vagueness in the US image using (intuitionistic and neutrosophic) fuzzy set theory [39]. Therefore, this research would definitely be an aid, even to experienced radiologists, by providing a second opinion for the characterization of nodules. Moreover, it could be used as a valuable tool in follow-up diagnosis (such as thyroid cancer) where the validity of conclusions drawn by radiologist depends on the delineation accuracy. Such techniques will help to aid the diagnosis process by automatically diagnosing the nodules in thyroid images and consequently lead to reduction of false diagnosis related thyroid diseases. As thyroid nodules were categorized as the enlarged follicles, the follicular cells with follicles, the papillary cells with follicles, the follicular cells with fibrosis, the papillary cells with fibrosis, and the fibrosis according to the pathology. Many physicians are confused about the nature of various echo patterns of thyroid nodules because of low resolution of ultrasound. The utilization of new and more efficient classifiers could improve the accuracy performance towards classifying thyroid nodule as benign/malignant. For increasing computational efficiency, extended fuzzy approaches, multiscale processing and parallelizable methods such as neural networks appear to be promising approaches.

Comparative studies of different feature extraction techniques and classification techniques should be carried out fairly, deeply and accurately. This paper basically provides a summary of existing automatic tools available to formulate the disease diagnosis part easier as well efficient. Different performance evaluation metrics are studied, and the future developments and trends are also investigated. Therefore, this work would definitely be an aid for future research as it provides an extensive study for an effective computer-aided diagnosis system of thyroid nodules in Ultrasound images. Moreover, it could be used as a valuable tool in follow-up diagnosis (such as thyroid cancer) where the validity of conclusions drawn by radiologist depends on the delineation accuracy. Such techniques will help to aid the diagnosis process by automatically detecting the nodules in thyroid images and consequently lead to reduction of false diagnosis related thyroid diseases.

The most urgent task is to build a benchmark database of US images accessible to the public to support the comparison and evaluation of different algorithms and CAD systems. Threedimensional ultrasound imaging is another future direction which has been paid more and more attention. Three-dimensional ultrasound imaging can provide more comprehensive information of the thyroid nodule than 2D imaging and incorporate all 2D characteristics. The advantages of 3D US are especially obvious in a CAD system because CAD system is good for processing a large amount of data in a short time, which can greatly reduced the variability of the observations and the work load of radiologists. Most of the 2D techniques can be directly applied to 3D images with some pre-processing or post-processing methods. Some newly developed methods, especially for 3D images, can be found in $[40,41]$. The optimal feature extraction and classification methods for thyroid nodule detection can be determined and the evolvement of the proposed time efficient scheme for application in an integrated real time system for the assessment of the thyroid gland in future. Future work could also involve the thyroid volume estimation from the segmented thyroid region and classification of thyroid nodules based on malignancy risk factor in ultrasound images.

\section{REFERENCES}

[1] Unnikrishnan A.G. and Menon U.V., "Thyroid disorders in India: An epidemiological perspective," Indian Journal of Endocrinology and Metabolism, Vol. 15, pp.78-81, Supplement 2, 2011. 
[2] Horvath E., Majlis S., Rossi R., Franco C., Niedmann J.P. and Castro A., "An ultrasonogram reporting system for thyroid nodules stratifying cancer risk for clinical management," J. Clin .Endocrinol Metab, pp.748-751, 2009.

[3] Ain K. and Rosenthal M.S., "The Complete Thyroid Book," Second Edition, 2011, Mc Graw Hill.

[4] Silver J. K., Parangi S. and Phitayakorn R., "Biographies of Disease," Series Editor, 2011.

[5] Baskin H.J., "Thyroid Ultrasound and Ultrasound-Guided FNA," Second Edition, springer, 2008.

[6] Kharchenko V.P., Kotlyarov P.M., Mogutov M.S., Alexandrov Y.K., Sencha A.N., Patrunov Y.N. and Belyaev D.V, "Ultrasound Diagnostics of Thyroid Diseases," Springer 2010.

[7] Sarti A., Corsi C., Mazzini E. and Lamberti C., "Maximum likelihood segmentation of ultrasound images with Rayleigh distribution," IEEE Trans. Ultrason. Ferroelect. Freq. Control, vol. 52, no. 6, pp. 947-960, 2005.

[8] Ma J., Luo S., Dighe M., Lim D. and Kim Y., "Differential Diagnosis of Thyroid Nodules with Ultrasound Elastography based on Support Vector Machines," IEEE Int. Ultrasonics Symp. Proc., pp.1372-1375, 2010.

[9] Savelonas M.A., Iakovidis D.K., Dimitropoulos N., and Maroulis D., "Computational Characterization of Thyroid Tissue in the Radon Domain", IEEE International Symposium on Computer-Based Medical Systems,pp.1-4, 2007.

[10] Chang C., Tsai M., and Chen S., "Classification of the Thyroid Nodules Using Support Vector Machines" 2008 International Joint Conference on Neural Networks (IJCNN 2008) pp.3093-3098.

[11] Temurtas F., "A comparative study on thyroid disease diagnosis using neural networks," Expert Systems with Applications 36, pp. 944-949, 2009.

[12] Keramidas E.G., Iakovidis D.K., Maroulis D. and Karkanis S., "Efficient and Effective Ultrasound Image Analysis Scheme for Thyroid Nodule Detection," ICIAR, LNCS 4633, pp. 1052-1060, Springer 2007.

[13] Savelonas M.A., Iakovidis D.K., Legakis I. and Maroulis D., "Active Contours Guided by Echogenicity and Texture for Delineation of Thyroid Nodules in Ultrasound Images," in IEEE Transactions on Information Technology in Biomedicine, vol. 13, pp.519-527, 2009.

[14] Morifuji, H., Analysis of ultrasound B-mode histogram in thyroid tumors. Nippon Geka Gakkai Zasshi 90(2):pp.210-221, 1989.

[15]Hirning, T., Zuna, I., and Schlaps, D., Quantification and classifica-tion of echographic findings the thyroid gland by computerized b-mode texture analysis. Eur J. Radiol 9:244-247, 1989.

[16] Saiti F., Naini A.A., Shoorehdeli M. A. and Teshnehlab M., "Thyroid Disease Diagnosis Based on Genetic Algorithms using PNN and SVM,"pp.1-4, 2009, IEEE.

[17] Keramidas E.G., Iakovidis D.K., Maroulis D. and Dimitropoulos N., "Thyroid Texture Representation via Noise Resistant Image Features," IEEE Int. Symp. on Computer-Based Medical Systems, pp.560-565, 2008.

[18] Shariati S. and Haghighi M.M., "Comparison of ANFIS neural network with several other ANNS and support vector machine for diagnosing hepatitis and thyroid diseases" In Int. Conf. on Computer Information Systems and Industrial Management Applications (CISIM), pp.596-599, 2010 IEEE.

[19] Chang C., Lei Y., Tseng C. and Shih S., "Thyroid Segmentation and Volume Estimation in Ultrasound Images," IEEE Int. Conf. on Systems, Man and Cybernetics (SMC 2008), pp.3442-3447.

[20]Tsantis S., Cavouras D., Kalatzis I., Piliouras N., Dimitropoulos N. and Nikiforidis G. "Development of A Support Vector Machine-Based Image Analysis system for assessing the Thyroid Nodule malignancy risk on ultrasound," Ultrasound in med. \& biol., vol. 31, no. 11, pp. 1451-1459, 2005.

[21] Seabra J.C.R. and Fred A.L.N., "Towards the Development of a Thyroid Ultrasound Biometric Scheme Based on Tissue Echo-morphological Features," in Springer-Verlag, BIOSTEC, CCIS 52, pp.286-298, 2010.

[22] Selvathi D. and Sharnitha V.S., "Thyroid Classification and Segmentation in Ultrasound Images Using Machine Learning Algorithms," In Proc. of Int. Conf. on Signal Processing, Communication, Computing and Networking Technologies, pp. 836-841, IEEE, 2011.

[23] Tsantis S., Dimitropoulos N., Cavouras D., Nikiforidis G., "Morphological and wavelet features towards sonographic thyroid nodules evaluation" Computerized Medical Imaging and Graphics 33 (20 09) 91-99

[24] Pal S.K., “A review on image segmentation techniques," Pattern Recognition 29, pp.1277-1294, 1993.

[25] Noble J.A. and Boukerroui D., "Ultrasound image segmentation: A survey," IEEE Trans. on Medical Imaging, 25, pp. 987-1010, 2006. 
[26] Keles A., "ESTDD: Expert system for thyroid diseases diagnosis," Expert Systems with Applications, pp.242-246, Elsevier, 2008.

[27] Dogantekin E., Dogantekin A. and Derya A., “An expert system based on Generalized Discriminant Analysis and Wavelet Support Vector Machine for diagnosis of thyroid diseases," Expert Systems with Applications, 38, pp.146-150, 2011.

[28] Koundal D., Gupta S. and Singh S., "Survey of Computer-Aided Diagnosis of Thyroid Nodules in Medical Ultrasound Images", The Second International Conference on Advances in Computing and Information Technology (ACITY 2012).

[29] Cheng H.D., Shan J., Ju W., Guo Y. and Zhang L., "Automated breast cancer detection and classification using ultrasound images: A survey," Pattern Recognition 43, pp.299-317, 2010.

[30] Joo S., Moon W.K. and Kim H.C. "Computer-aided diagnosis of solid breast nodules on ultrasound with digital image processing and artificial neural network," In IEEE Int. Conf. Proc. on Engg. in Medicine and Biology Society, pp.1397-13400, 2004.

[31]Fu K.S. and Mui J.K., “A survey on image segmentation,” Pattern Recognition, 131, pp.3-16, 1981.

[32]Zhang G., Berardi V. L., "An investigation of neural networks in thyroid function diagnosis" Health Care Management Science 1 (1998) pp.29-37.

[33] Savelonas M.A., Maroulis D.E., Iakovidis D.K. and Dimitropoulos N., "Computer-Aided Malignancy Risk Assessment of Nodules in Thyroid US Images Utilizing Boundary Descriptors," in Panhellenic Conf. on Informatics, pp.156-160, 2008, IEEE.

[34] Shukla A., Kaur P., Tiwari R. and Janghel R.R., "Diagnosis of Thyroid Disorders using Artificial Neural Networks,” IEEE Int. Advance Computing Conf., pp.1016-1020, 2009.

[35] Rouhani M. and Mansouri K., "Comparison of several ANN architectures on the Thyroid diseases grades diagnosis," Int. Association of Computer Science and Information Technology-Spring Conf., pp.526-528, 2009, IEEE.

[36] Polat K., Sahan S. and Gunes S., "A novel hybrid method based on artificial immune recognition system (AIRS) with fuzzy weighted pre-processing for thyroid disease diagnosis," Expert Systems with Applications 32, pp.1141-1147, 2007.

[37] Kodaz H., Seral O., Arslan A. and Salih G., "Medical application of information gain based artificial immune recognition system (AIRS): Diagnosis of thyroid disease," Expert Systems with Applications, pp.3086-3092, 2009.

[38]Chang C.Y., Lei Y., Tseng C. and Shih S., "Thyroid Segmentation and Volume Estimation in Ultrasound Images," In IEEE Transactions on Biomedical Engineering, vol. 57, pp. 1348-1357, 2010.

[39] Koundal D., Gupta S. and Singh S., "Applications of Neutrosophic and Intuitionistic Fuzzy Set on Image Processing" in National Conference on Green Technologies: Smart and Efficient Management (GTSEM-2012).

[40]Chuan-Yu Chang and Yong-Cheng Hong, "A Neural Network for Thyroid Segmentation and Volume Estimation in CT Images" November 2011, IEEE Computational Intelligence Magazine, pp 43-55.

[41]F. Molinari, A. M. vani, M. Deandra, P. Limone, R. Acharya, R. Garberogio, and J. S "Skeletonization of 3D Contrast Enhanced Ultrasound Images for the Characterization of Single Thyroid Nodule" A. El-Baz et al. (eds.), Multi Modality State-of-the-Art Medical Image Segmentation and Registration Methodologies, pp 137-159.

\section{Authors}

D. Koundal received the B. Tech degree from SKIET, kurukshetra university, Haryana in 2004, and the M.E Degree from UIET, Panjab University, Chandigarh in 2009. Also pursuing full time Ph.D. at UIET, PU, Chandigarh. Her research interests include Image pre-processing, segmentation and classification.

S. Gupta received the B.Tech. degree from TITS, Bhiwani, in 1992, and the M.E. degree from TIET, Patiala, Punjab, in 1998, both in Computer Science and Engineering. She has been teaching and involved in research activities since 1992. She obtained her Ph. D. Degree from PTU, Jalandhar in 2007 in the field of Ultrasound image processing. Presently, she is working as Professor in the Department of Computer Science \& Engineering, University Institute of Engineering \& Technology, Panjab University, Chandigarh. Her research interests include wavelet based image processing, network security, image compression and denoising. 
International Journal of Computer Science \& Engineering Survey (IJCSES) Vol.3, No.4, August 2012

S. Singh obtained his B.Tech. (Computer Engg.) degree from GNDU Amritsar (Punjab) in 1991 and M.E (Computer Science \& Engg.) Hons. degree from Thapar Institute of Engineering and Technology, Patiala in 1999. He obtained his Ph.D. degree from IIT Roorkee in 2005 in the field of Medical Image Processing. He has been in the teaching profession since 1992 and has published more than 40 papers in refereed International Journals and Conference Proceedings. Presently he is serving as Professor in the Department of CSE, University Institute of Engg. \& Technology, Panjab University, Chandigarh. He is the life time member of ISTE, member of other professional bodies. His research interests include Medical Image Compression and Analysis, Telemedicine and Network Security. 